\title{
Nihil nocere, auch in der Sportmedizin
}

Im Hinblick auf den vom Parlament Ende 2000 beschlossenen Dopingartikel im Bundesgesetz über Turnen und Sport [1] hat eine Arbeitsgruppe der Sportmediziner auf Wunsch der FMH eine kurze Ergänzung der Standesordnung und eine möglichst praxisbezogene Auslegeordnung der rechtlichen und berufsethischen Fragen erarbeitet, die sich bei der sportmedizinischen Betreuung von Athleten und Freizeitsportlern stellen. Der Entwurf war im Sommer 2001 in Vernehmlassung [2, 3] und stiess auf ein positives Echo. Die Ärztekammer konnte das Geschäft im Dezember 2001 aus Zeitmangel nicht mehr behandeln, sie hat dem Entwurf jedoch im April 2002 diskussionslos zugestimmt. Nach Ablauf der Referendumsfrist treten

- ein Sportmedizinartikel in der FMH-Standesordnung (Art 33bis),

- die Anpassung einiger bestehender Bestimmungen der Standesordnung (betrifft Art. 6, 7 und 33) sowie

- die Richtlinie mit Verhaltensregeln zur Sportmedizin (Anhang 5 zur Standesordnung)

mit der heutigen Publikation in Kraft.

1 Der Gesetzestext ist publiziert im Bundesblatt vom 28. Dezember 2000, S. 6115ff

(im Zusammenhang mit dem Bundesgesetz über Arzneimittel und Medizinprodukte). http://www.admin.ch/ch/d/ff/2000/6115.pdf.

2 Monnat A. Sportmedizin in der FMH-Standesordnung. Schweiz Ärztezeitung 2001;82(31):1642-5.

3 Sportmedizin und Standesrecht. Schweiz Ärztezeitung 2001;82(31):1646-50.

\section{Richtlinien für die ärztliche Betreuung von Sportlern und Sportlerinnen ${ }^{1}$}

\section{Präambel}

1 Ärzte und Ärztinnen leisten durch Information, Beratung und Betreuung ihrer Patienten und Patientinnen im Hinblick auf sportliche Tätigkeiten einen wesentlichen Beitrag zur Gesundheitsförderung.

2 Der Sport hat eine nationale Bedeutung. Spitzenathleten und -athletinnen stehen oft unter dem Druck von Öffentlichkeit, Presse, Trainern und Verbandsorganen, Sponsoren und ihrer selbst, immer bessere Leistungen zu erbringen. Athleten und Athletinnen sind gefordert, an ihre Grenzen zu gehen. Dies kann zu gesundheitlichen Problemen führen, Risiken mit sich bringen und unlautere Wettbewerbsbedingungen hervorrufen.

3 Die sportmedizinische Betreuung von Athletinnen und Athleten verlangt viel Verständnis. Sie setzt ein verantwortungsbewusstes Handeln für die Gesundheit der Sporttreibenden innerhalb der Grenzen der ärztlichen
Ethik voraus und soll die Fairness im sportlichen Wettkampf aufrechterhalten. Dazu gehört auch die Einsicht, dass primär nicht Arzt oder Ärztin für die Entwicklung der sportlichen Leistung verantwortlich sind, und diese sich nicht ungebührlich über sportliche Erfolge profilieren sollen.

\section{Geltungsbereich}

1 Diese Richtlinie gilt sowohl für die ärztliche Beratung und Betreuung von «Sporttreibenden im Allgemeinen» wie auch für die sportmedizinische Tätigkeit von Ärzten und Ärztinnen bei der Beratung und Behandlung von lizenzierten Athleten und Athletinnen eines dem Swiss Olympic angeschlossenen Sportverbandes oder Vereins im Besonderen.

2 «Sporttreibende im Allgemeinen» sind sowohl Kinder als auch Jugendliche und Erwachsene, die sich sportlich betätigen und 
im Hinblick auf diese Tätigkeit ärztlichen Rat suchen, sowie Teilnehmende am «nicht reglementierten Wettkampfsport».

3 Athleten und Athletinnen im Besonderen sind sämtliche Teilnehmer am «reglementierten Wettkampfsport». Unter den Begriff «reglementierter Wettkampfsport» fallen (in Analogie zum Dopingstatut des SOV vom 1.1.2000, Art. 13) Sportanlässe, die von einem bei Swiss Olympic angeschlossenen Verband oder Verein oder deren internationalen Dachverbänden organisiert werden, sowie die Vorbereitung durch Training oder Regeneration darauf.

\section{Sportmedizinische Grundsätze}

\subsection{Gesundheitsschutz und Patienten-} autonomie: ein potenzieller Zielkonflikt

1 Im Vordergrund jeder sportmedizinischen Tätigkeit von Ärzten und Ärztinnen stehen die Überwachung und der Schutz der Gesundheit der Sporttreibenden. Wichtiges Prinzip ist bei jeder Entscheidung sowohl im medizinischen wie im ethischen und rechtlichen Sinn das «primum nihil nocere». Ärzte und Ärztinnen, die Sporttreibende betreuen, berücksichtigen das Können, das Engagement und die oft aussergewöhnliche physische und psychische Belastung, die durch sportliche Leistungen abverlangt werden.

2 Athleten und Athletinnen sind häufig hochmotiviert oder stehen unter Druck, die sportliche Tätigkeit trotz gesundheitlicher Risiken gegen ärztliche Empfehlungen weiterzuführen.

3 Falls die Teilnahme am Training oder an Wettkämpfen mit der Erhaltung der Gesundheit eines oder einer Sporttreibenden nicht zu vereinbaren ist, aber der oder die Sporttreibende auf einer Teilnahme beharrt, so liegt für Arzt und Ärztin ein berufsethischer Zielkonflikt vor (Gesundheitsschutz versus Patientenautonomie).

\subsection{Kriterien für den Umgang mit diesem Zielkonflikt}

Es ist zu unterscheiden bzw. abzuwägen,

- ob der Arzt oder die Ärztin ausschliesslich vom oder von der Sporttreibenden beauftragt ist («Privatarzt oder Privatärztin») oder sportmedizinische Funktionen im Rahmen des organisierten Sports erfüllt (wie Verbands-, Club-, Mannschafts- oder Teamarzt, im nachfolgenden alle «Teamarzt und Teamärztin» genannt);
- wie gross die gesundheitlichen Nachteile oder Risiken sind;

- wie weitgehend der oder die Sporttreibende die vom Arzt oder von der Ärztin durchgeführte Aufklärung über die Nachteile und Risiken verstehen (Entscheidungsfähigkeit);

- wie weitgehend der oder die Sporttreibende ihre Entscheide frei und unbeeinflusst von äusseren Zwängen, insbesondere von Team, Trainer, Familie, sportlicher Karriere und Einkommen, fällen können (Entscheidungsfreiheit).

\subsection{Kriterien für fehlende Entscheidungs- fähigkeit oder -freiheit des oder der Sport- treibenden}

Insbesondere in folgenden Fällen können die Entscheidungsfähigkeit oder -freiheit von Sporttreibenden eingeschränkt sein oder ganz fehlen:

- Situationen, in denen der oder die Sporttreibende nicht fähig ist, vernünftig zu entscheiden (z.B. Bewusstseinsstörungen, epileptische Anfälle, reaktive Psychosen);

- Jugendliche unter 16 Jahren (nachfolgende Ziff. 3.6.);

- Sportler und Sportlerinnen, die in einem faktischen Arbeitsverhältnis stehen (nachfolgende Ziff. 3.5.).

\subsection{Verhaltensempfehlung für Privatarzt, -ärztin}

1 Werden Arzt oder Ärztin ausschliesslich im Auftrag des oder der Sporttreibenden tätig, raten sie diesem oder dieser mit der gebotenen Klarheit von einer sportlichen Tätigkeit $\mathrm{ab}$, die mit der Erhaltung seiner oder ihrer Gesundheit nicht zu vereinbaren ist.

2 Sie attestieren keine Trainings- oder Wettkampftauglichkeit, die sie mit ihrem Gewissen nicht vereinbaren können (vgl. Art. 3 Abs. 4 StaO).

3 Im Verhältnis zu Dritten respektieren sie das Patientengeheimnis des oder der Sporttreibenden (vgl. Art. 11 StaO). Vorbehalten bleiben die Bestimmungen für die Betreuung von Kindern und Jugendlichen (nachfolgende Ziff. 3.6.) sowie die Fälle, in denen der oder die Sporttreibende die Gesundheit Dritter gefährden (nachfolgende Ziff. 3.8.).

\subsection{Verhaltensempfehlung für Teamarzt, -ärztin}

1 Erfolgt die medizinische Beurteilung eines oder einer Sporttreibenden im Auftrag eines Dritten (Verband, Teamverantwortlicher, etc.) müssen sich Teamarzt und -ärztin des Interessenkonfliktes bewusst sein, der zwischen dem 
oder der Sporttreibenden und dem Auftraggeber bestehen kann (vgl. Art. 33 StaO).

2 Bei der Übernahme einer Verpflichtung als Teamarzt oder -ärztin sichert sich dieser oder diese vertraglich ein Melderecht für die Bekanntgabe von Sporttauglichkeitsentscheiden an den Auftraggeber zu.

3 Bei der Beurteilung der Sporttauglichkeit eines oder einer Sporttreibenden für den Auftraggeber müssen Teamarzt oder -ärztin abwägen zwischen den gesundheitlichen Risiken und der Frage, wie weit Entscheidungsfähigkeit und -freiheit des oder der Sporttreibenden gegeben sind, die trotz gesundheitlicher Risiken teilnehmen möchten (vgl. Art. 3 Abs. 4 und Art. 4 Abs. 1 StaO). Teamarzt oder -ärztin attestieren keine Trainingsoder Wettkampftauglichkeit, die sie mit ihrem Gewissen nicht vereinbaren können (vgl. Art. 3 Abs. 4 StaO).

4 Steht der oder die Sporttreibende in einem faktischen Arbeitsverhältnis zu einem Verband, einem Team etc., und bestehen Zweifel an seiner oder ihrer Entscheidungsfähigkeit oder -freiheit und ist ein Einsatz in Training oder Wettkampf aus ärztlicher Sicht nicht vertretbar, gewichten und entscheiden Teamarzt oder -ärztin den Zielkonflikt zwischen Gesundheitsschutz und Patientenautonomie. Sie berücksichtigen dabei auch die Fürsorgepflicht des Arbeitgebers gegenüber dem Arbeitnehmer. Nötigenfalls ergreifen sie die Initiative und weisen die zuständigen bzw. geeigneten Personen bzw. Stellen auch gegen den Willen des oder der Sporttreibenden auf dessen oder deren Trainings- oder Wettkampfuntauglichkeit hin.

5 Teamarzt oder -ärztin sollen den oder die Sporttreibenden vor Untersuchungen darüber informieren, aus welchem Grund die Untersuchung durchgeführt wird, und wer über die Schlussfolgerungen zu informieren ist. Das weitere Vorgehen und gegebenenfalls der Inhalt einer notwendigen Mitteilung an Dritte werden mit dem oder der Sporttreibenden nach der Untersuchung besprochen.

6 Informationen von Teamarzt oder -ärztin an den Auftraggeber betreffen in jedem Fall ausschliesslich die sportmedizinischen Schlussfolgerungen, also eine - nötigenfalls differenzierte - Beschreibung der Trainingsbzw. Wettkampf(un)tauglichkeit, nicht aber deren medizinische Begründung (keine Angabe der Diagnose; vgl. Art. 11 in Verbindung mit Art. 33 StaO).

7 In jedem Fall ist bei Gefährdung Dritter nachfolgende Ziff. 3.8. zu beachten.

\subsection{Kinder und Jugendliche}

1 Handelt es sich bei dem oder der Sporttreibenden um ein Kind, einen Jugendlichen oder eine Jugendliche, müssen Arzt und Ärztin insbesondere sicherstellen, dass die Anforderungen des Trainings und der Wettkämpfe dem Wachstum und der Entwicklung entsprechen und die normale körperliche und geistige Entwicklung nicht gefährden.

2 Das Kind, der Jugendliche oder die Jugendliche sollen auf eine für sie verständliche Weise über das Vorgehen aufgeklärt werden.

3 Gesundheitliche Probleme müssen gegebenenfalls auch mit den Eltern (oder einem allfälligen anderen gesetzlichen Vertreter) besprochen werden.

4 Das Fällen eines Entscheides wird massgeblich vom individuellen Entwicklungsstand und von der zur Entscheidung anstehenden Frage beeinflusst. Im Sinne einer Faustregel kann festgehalten werden, dass bei Kindern und Jugendlichen zwischen 12 und 16 Jahren sportmedizinische Entscheidungen gemeinsam mit den Eltern (oder einem allfälligen gesetzlichen Vertreter) getroffen werden. Bei Kindern unter 12 Jahren kann die Fähigkeit zur Mitbestimmung nur in Ausnahmefällen angenommen werden ${ }^{2}$.

\subsection{Schweigepflicht und Öffentlichkeit}

1 Gegenüber der Öffentlichkeit halten Arzt und Ärztin die Schweigepflicht (Patientengeheimnis) ein.

2 Handelt es sich um einen Athleten oder eine Athletin, dessen oder deren Gesundheitszustand von öffentlichem Interesse ist, soll der Arzt oder die Ärztin zusammen mit dem Athleten oder der Athletin entscheiden, welche Informationen an die Öffentlichkeit gelangen sollen. Wo es die Umstände erlauben, soll gemeinsam ein schriftliches Communique abgegeben werden, um Missverständnisse zu vermeiden.

\subsection{Gefährdung Dritter}

1 Besteht aus ärztlicher Sicht eine Gefährdung Dritter (wie z.B. Spielende des eigenen oder gegnerischen Teams, Publikum), so soll der behandelnde Arzt oder die behandelnde Ärztin den Sporttreibenden oder die Sporttreibende darüber aufklären und von der Teilnahme abraten. Falls nötig, soll der Arzt oder die Ärztin die zuständigen bzw. geeigneten Personen oder Stellen auch gegen den Willen des oder der Sporttreibenden auf dessen oder deren Trainings- und Wettkampfuntauglichkeit hinweisen. einteilung erscheint auch für die Sportmedizin als sachgerecht. 
2 Wenn kein vertraglich festgelegtes Melderecht besteht und der oder die Sporttreibende das Einverständnis zur aus ärztlicher Sicht notwendigen Information den zuständigen bzw. geeigneten Personen oder Stellen verweigert, ist vorgängig die Entbindung vom ärztlichen Berufsgeheimnis (Patientengeheimnis) bei der zuständigen kantonalen Behörde einzuholen (Art. 321 StGB). Nur wenn eine unmittelbare, nicht anders abwendbare grosse Gefahr für die Gesundheit Dritter besteht, darf die Information gegen den Willen des oder der Sporttreibenden ohne behördliche Entbindung vom Berufsgeheimnis erfolgen (Art. 32 Ziff. 2 StGB, Notstandshilfe ${ }^{3}$.

\section{Doping im «reglementierten Wettkampfsport»}

\subsection{Definition}

1 Doping im Sinne dieser Richtlinien betrifft den «reglementierten Wettkampfsport». Die nachfolgende Definition hält sich an die Umschreibung der Olympischen Bewegung im Antidoping-Code (gültig ab 1.1.2000) ${ }^{4}$. Diese lautet wie folgt: Doping widerspricht den fundamentalen Prinzipien des Olympischen Sports und der medizinischen Ethik und ist somit verboten. Doping ist die Verwendung von Hilfsmitteln in Form von Substanzen oder Methoden, welche potenziell gesundheitsschädigend sind und/oder die körperliche Leistungsfähigkeit steigern können. Doping ist aber auch das Vorhandensein einer verbotenen Substanz im Körper einer Sportlerin oder eines Sportlers oder die Bestätigung deren Verwendung oder der Verwendung einer verbotenen Methode.

2 Seine Anwendung kann der Gesundheit der Sporttreibenden schaden, verstösst gegen die Grundsätze der ärztlichen Ethik und ist im Rahmen sportlicher Wettbewerbe unfair. Daher ist im «reglementierten Wettkampfsport» das Herstellen, Einführen, Vermitteln, Vertreiben, Verschreiben, Abgeben und Überwachen von Doping durch Arzt und Ärztin standesunwürdig und unzulässig ${ }^{5}$.

\subsection{Dopingliste}

1 Für die Bezeichnung der verbotenen Substanzen und Massnahmen ist die Dopingliste von Swiss Olympics in der jeweils aktuellen Fassung $^{6}$ und/oder die Dopingverordnung des VBS $^{7}$ massgebend. Jede Substanz, die einer der verbotenen Substanzklassen angehört, gilt als Doping, auch wenn sie nicht namentlich aufgeführt ist. Dies bezieht sich auf die Verwandtschaft bezüglich der pharmakologischen Wirkung wie der chemischen Struktur. Zudem ist der Erfolg oder Misserfolg bei der Verwendung einer verbotenen Substanz oder einer verbotenen Methode unerheblich. Es genügt die blosse Verwendung/Anwendung oder der Versuch einer Verwendung/Anwendung, um einen Dopingverstoss zu begehen.

2 Die folgenden aufgezählten Mittel und Methoden sind bei Erlass der vorliegenden Richtlinie in der Dopingliste enthalten:

- Die Anwendung von Medikamenten oder anderen Substanzen, die den folgenden Klassen angehören: Stimulanzien, Narkotika, Anabolika, Diuretika, Peptidhormone und analog wirkende Substanzen (Mimetics).

- Die Anwendung der folgenden Substanzklassen untersteht gewissen Einschränkungen: Alkohol, Cannabinoide, Lokalanästhetika, Kortikosteroide, Betablocker.

- Pharmakologische, chemische und physikalische Manipulationen, welche die Integrität einer Dopingkontrollprobe (z.B. Urin, Blut) beeinflussen. Dies beinhaltet z.B. Austausch, Verdünnung oder anderes Beeinflussen der renalen Stoffausscheidung, Beeinflussung des Verhältnisses von Testosteron zu Epitestosteron.

- Die Anwendung von Blutdoping, d.h. die Gabe von Erythrozyten, künstlichen Sauerstoffträgern, Plasmaexpandern oder von analogen Produkten.

\subsection{Zwingende medizinische Indikation, Bewilligung durch die Fachkommission für Dopingbekämpfung (FDB)}

1 Falls der Arzt oder die Ärztin, die wissen, dass es sich um einen Athleten oder eine Athletin handelt, die Verabreichung eines Medikamentes mit verbotenen Substanzen oder die Anwendung einer verbotenen Methode mangels adäquater Alternativen und aus zwingenden medizinischen Gründen als notwendig erachten, haben sie vorgängig die Einwilligung des Vertrauensarztes oder der Vertrauensärztin ${ }^{8}$ der Fachkommission für Dopingbekämpfung $\left(\mathrm{FDB}^{9}\right)$ von Swiss Olympic einzuholen. Die Einwilligung kann befristet und mit Auflagen bezüglich der Teilnahme an Wettkämpfen verbunden werden.

2 Vorbehalten bleiben lebenserhaltende Sofortmassnahmen. Diese sind unverzüglich der FDB zu melden. 
3 Eine Behandlung mit anabol-androgenen Substanzen ist im Prinzip nicht gestattet. Für seltenste Fälle, in denen eine hormonelle Substitution beim Vorliegen einer nachgewiesenen gonadalen Unterfunktion indiziert ist, braucht es die befristete und mit Auflagen bezüglich der Teilnahme an Wettkämpfen versehene Einwilligung der FDB.

\subsection{Aufklärung, Zusammenarbeit, Abwehr von Druck}

Der Arzt und die Ärztin müssen die Sporttreibenden, ihre Verbandsorgane und andere Betroffene über die Gründe und Konsequenzen der Ablehnung von Dopingmethoden aufklären, die Kontrollorgane unterstützen und nach Möglichkeit die Sporttreibenden vor jeglichem Druck von aussen schützen, mit dem diese zu solchen Methoden gezwungen werden könnten.

\section{Medikamentenmissbrauch bei «Sporttreibenden im Allgemeinen»}

1 Wenn «Sporttreibende im Allgemeinen» aus Gründen der Leistungssteigerung Substanzen und Methoden anwenden, die im «reglementierten Wettkampfsport» gemäss Ziff. 4 als Doping gelten, liegt ein Medikamentenmissbrauch vor. Arzt und Ärztin befinden sich hier in einem Dilemma zwischen den Grundsätzen ihrer Berufsausübung (Art. 2 $\mathrm{StaO}$ ) und den individuellen Bedürfnissen der Sporttreibenden, die sie beraten oder behandeln. Obschon der Verzicht auf diesen Medikamentenmissbrauch das ideale Ziel einer Beratung oder Behandlung ist, kann er nicht immer zur Bedingung für deren Gewährung gemacht werden.

2 Für den Umgang mit diesem Dilemma des Medikamentenmissbrauchs bei der Beratung und Behandlung von «Sporttreibenden im Allgemeinen» sollten folgende Ziele als Orientierungshilfen dienen.

- Medikamente und Methoden, die auf der Dopingliste stehen, sollten nicht oder nur mit äusserster Zurückhaltung abgegeben oder angewendet werden.

- Wenn verbotene Substanzen ${ }^{10}$ abgegeben werden, weil keine Behandlungsalternativen bestehen, ist eine vollumfängliche Orientierung der Sporttreibenden erforderlich.

10 Abrufbar über www.dopinginfo.ch; Doping; Dopingliste.

\section{Präventive Massnahmen}

1 Eine wirksame Prävention von Doping und Medikamentenmissbrauch muss sich auf eine gute Zusammenarbeit zwischen den Sporttreibenden, den Sportverbänden, deren Dachverband, den zuständigen Bundesstellen und den beteiligten Ärzten und Ärztinnen stützen.

2 Sie steuert der Einstellung entgegen, sportliche Leistungen durch künstliche Mittel steigern zu wollen. Sie besteht aus Information und Aufklärung bereits bei sporttreibenden Kindern und Jugendlichen und setzt sich später bei der individuellen ärztlichen Beratung aller Sportler und Sportlerinnen fort (Vergleiche Ziff. 4 und 5). Insbesondere zeigt sie als Alternative zu Doping und Medikamentenmissbrauch die Vorteile des gewissenhaften Trainingsaufbaus.

3 Die Information darf nicht banalisierend sein und beinhaltet auch, dass Doping und Medikamentenmissbrauch im Sport nicht nur ein individuelles Problem sind, sondern die Gründe auch in der Gesellschaft zu suchen sind.

4 Daher unterstützen Arzt und Ärztin auch Massnahmen zur Beschränkung des Zuganges zu Dopingsubstanzen und Methoden.

\section{Inkraftsetzung}

Die Richtlinie und die revidierten Art. 6, 27, 33 Standesordnung sowie der neu eingefügte Art. 33bis Standesordnung treten mit der Publikation in der Ärztezeitung in Kraft.

\section{Erläuterungen (Standesordnung und Richtlinie)}

Doping als «die beabsichtigte oder unbeabsichtigte Verwendung von Wirkstoffen aus verbotenen Substanzklassen und von verbotenen Methoden», wie in der Definition des Internationalen Olympischen Komitees IOK festgehalten (wobei natürlich als Ziel dieser verbotenen Verwendung die sportliche Leistungssteigerung gemeint ist), ist nicht länger nur eine Angelegenheit von Sportärzten, sondern betrifft als Phänomen und Spiegelung gesellschaftlicher Vorgänge die Ärzteschaft als Ganzes. Persönlicher sportlicher Ehrgeiz, nationales Prestige, finanzielle Verlockungen und ärztlicher Erfolgszwang können in diesem Bereich bedenkliche Partnerschaften eingehen. 
Bundesrat und Parlament haben dies erkannt und mit einer Änderung des Bundesgesetzes zur Förderung von Turnen und Sport aus dem Jahr 1972 Massnahmen gegen das Doping für verbindlich erklärt. Diese sollen die Dopingprävention fördern, die verbotenen Handlungen definieren, Kontrollen institutionalisieren und schliesslich Strafbestimmungen für Herstellung, Vertrieb, Abgabe und Anwendung von Dopingmitteln und -methoden formulieren, die bis $\mathrm{zu}$ Bussen von Fr. 100 000.- gehen. Damit wird auch Konformität mit der von der Schweiz bereits 1993 ratifizierten Europaratskonvention gegen Doping erreicht.

Bemerkenswert an diesen Strafbestimmungen ist, dass nicht der Konsument von Doping, also der Sportler, bestraft werden soll, sondern dessen «Umfeld», worunter der betreuende-und anwendende - Trainer- und Ärztestab verstanden wird. Die Sanktionierung der Sportler selbst fällt seit dem 1.1.2002 in die Zuständigkeit einer zentralen Strafbehörde unter dem Dach von Swiss Olympic.

Wichtig zum Verständnis der ganzen Problematik ist, dass sich die Schweizer Gesetzgebung auf den definierten Sektor des sogenannten «reglementierten Wettkampfsports» bezieht, nicht aber den Sport in seiner gesamten Breite erfasst. Für letzteren braucht es vom Gesetzgeber unabhängige ärztliche Überlegungen und Verhaltensanweisungen, die selbstverständlich in die gleiche Richtung zu zeigen haben.

Die skizzierte Rechtssituation erfordert zwingend, dass jeder Arzt in der Schweiz weiss,

- dass eine Dopingliste existiert, und

- dass die Abgabe sowie Verschreibung von Medikamenten dieser Liste an Athleten des reglementierten Wettkampfsports strafbar ist.

So wird unter Umständen bereits die unbedachte Abgabe eines zusammengesetzten Grippemittels (z. B. ein Stimulans enthaltend) im reglementierten Wettkampfsport zu unangenehmen Nachfragen, Untersuchungen oder gar strafrechtlichen Folgen führen können. Andere Beispiele wie spektakuläre Situationen um vermutete oder bewiesene Nachhilfe durch Einsatz von Erythropoietin EPO im Ausdauersport oder um den Anabolika-Schwarzmarkt für Körperformung und -kräftigung sind aus der Presse hinreichend bekannt. Neben Medikamentenfragen können aber auch zahlreiche andere Konstellationen die Beziehung zwischen einem sportwilligen Patienten oder Klienten und einem beispielsweise seine Sporttauglichkeit in Frage stellenden Arzt vor schwierige Entscheide stellen.
Die Arbeitsgruppe schlägt vor, die grundsätzliche Unzulässigkeit des Dopings in einem einzigen Artikel 33bis der Standesordnung festzuschreiben (die FMH-Standesordnung selbst soll kurz und prägnant sein). Zudem werden die bestehenden Artikel der Standesordnung, die sich auf die klassischen «Dreiecksverhältnisse» beziehen (Vertrauensarzt, Arbeitsmediziner, Schularzt etc.), um die Sportmedizin ergänzt. Dies betrifft die Art. 6, 27 und 33 der Standesordnung.

Als konkreter Anwendungskommentar zu dieser Kurzformulierung wird zusätzlich in einem Anhang (5) der Standesordnung FMH eine ebenfalls von der Ärztekammer beschlossene Richtlinie für die ärztliche Betreuung von Sportlern aller Kategorien angefügt. Diese befasst sich nicht nur mit dem Phänomen und zweifellosen Hauptproblem Doping, sondern geht auch auf andere schon angetönte Konfliktmomente im Betreuungsverhältnis Arzt - Sportler ein, zu dem oft als dritter und nicht zu vernachlässigender Partner der «Arbeitgeber» in Form eines Sportclubs o. ä. tritt. Es kann nämlich einen ganz wesentlichen Unterschied bedeuten, ob der Arzt in einer privatärztlichen oder aber von dritter Seite beauftragten Funktion (sog. Teamarzt) mit dem Sportler in Kontakt steht. Die Richtlinie soll auch in dieser oft problembeladenen Zone, wo sich persönlich-autonome Absichten eines Sportlers, medizinische Bedenken des Arztes und pragmatische Vorstellungen der arbeitgebenden Sportinstitution kreuzen können, eine Hilfestellung bieten. Besonders bemerkenswert ist in diesem Zusammenhang die Formulierung eines ärztlichen Melderechts dem Auftraggeber gegenüber, ohne dessen vertragliche oder reglementarische Fixierung eine sinnvolle verbandsärztliche Tätigkeit nach Auffassung der Kommission nur unbefriedigend erfüllt werden kann.

Häufig stellt sich auch die Frage der Öffentlichkeitsinformation via Medien. Für die Arbeitsgruppe ist eindeutig, dass die Öffentlichkeit zwar einen Wunsch nach, aber kein Anrecht auf Information über das Arzt-Sportler-Verhältnis bzw. die medizinische Lage haben darf. In bestimmten Fällen mag es opportun sein, über Details zu informieren. Stets hat der Sportler dabei aber sein klares Vetorecht (Richtlinie 3.7).

Die Hauptaussagen des neuen Standesordnungsartikels 33bis sind klar: Doping im Rahmen der ärztlichen Tätigkeit ist unzulässig, und Medikamentenmissbrauch muss auch im nicht reglementierten Sport durch Arzt und Ärztin soweit möglich verhindert werden.

Massgebend ist (trotz Kritik wegen Unbestimmtheit der allgemeinen Formulierung im 
11 Vgl. den analogen Positionsbezug im Drogenkonzept der FMH: Grundsätzliche Überlegungen zur Drogenpolitik - die Rolle der Ärzteschaft, Schweiz Ärztezeitung 1996;9:349-57. «Abstinenz von illegalen Drogen ist das ideale Ziel einer Behandlung. Sie kann aber nicht immer zur Anfangsbedingung gemacht werden, um Zugang zu einer Behandlung zu gewähren.» (Schlussfolgerung von Ziffer 2.4. S. 355)

12 Das Sportrecht betont die hohe Eigenverantwortung des am reglementierten Wettkampfsport teilnehmenden Athleten, der sich beispielsweise «vor Einnahme eines Medikamentes vergewissern [muss], dass sich keine verbotenen Substanzen im Arzneimittel befinden.» (Krähe C. Beweislastprobleme bei Doping im internationalen Sport. In: Fritzweiler J. [Hrsg.] Doping. Bern, München und Wien: Verlage Stämpfli, Beck und Manz; 2000, S. 52.) ersten Absatz der Bestimmung) die Dopingdefinition der Olympischen Bewegung (in der Schweiz vertreten durch Swiss Olympic). Sie bezieht und grenzt sich, wie eingangs erwähnt, auf den reglementierten Wettkampfsport ein (Abschnitt 4. «Doping» der vorstehenden Richtlinie). Deshalb musste für das ärztliche Standesrecht ein Modus gefunden werden, der auch im quantitativ wesentlich bedeutenderen Breitensport für klare Verhältnisse sorgt. Bei dieser im Abschnitt 5. «Sporttreibende im Allgemeinen» angesprochenen grossen Population ist richtigerweise das Strafrecht nicht anwendbar, doch ist ebenso einleuchtend, dass auch da soweit möglich verhindert werden soll, was im Wettkampfsport unter Strafe steht.

Aus diesem Grund wird neu, in Analogie zum Dopingbegriff, der Begriff des «Medikamentenmissbrauchs» eingeführt, den in der Sportlerbetreuung involvierte Ärztinnen und Ärzte nach Möglichkeit zu verhindern oder - in der Realität oft wohl zutreffender - einzuschränken suchen sollen. Die Kategorisierung von strafbarem Doping und bloss gerügtem Medikamentenmissbrauch bedeutet nicht zweierlei Recht für Sporttreibende. Sie entspricht der einzig rationalen Lösung, den Sport als eine der «schönsten Nebensachen der Welt» an seiner Spitze möglichst unmanipuliert, fair und «sauber» zu erhalten, und gleichzeitig im übrigen Sport entsprechende Zeichen zu setzen. Abstinenz soll dabei im Breitensport, ähnlich wie in der Bekämpfung von Alkohol-, Nikotin- und anderen Suchtmittelabhängigkeiten, das ideale Ziel darstellen, wird aber nicht in jedem Fall die unbedingte Behandlungsvoraussetzung sein können. ${ }^{11}$

Mit dem parallel zum Dopingbegriff formulierten «Medikamentenmissbrauch bei Sporttreibenden im Allgemeinen» wird klargestellt, dass die Medikamentenabgabe an Gesunde, insbesondere an Sporttreibende, grundsätzlich sehr kritisch zu betrachten ist. Auch bei behandlungsbedürftigen Sporttreibenden im Allgemeinen sollen Medikamente möglichst restriktiv und nach einem Kontrollblick auf die Dopingliste von Swiss Olympic eingesetzt werden. In Einzelfällen können sich scheinbar verwirrliche Nuancen ergeben: das schon angesprochene ephedrinhaltige Grippemittel bedeutet wegen seiner denkbaren leistungssteigernden Potenz auch wenn nicht in dieser Absicht verabreicht beim lizenzierten Sportler Doping, beim Breitensportler Medikamentenmissbrauch, beim «Normalverbraucher» ist es unbedenklich. Entscheidend ist damit die Frage, ob der am reglementierten Wettkampfsport teilnehmende Athlet seinen Status dem Arzt mitteilen oder ob der Arzt seinen Patienten danach fragen muss. Aus Sicht des Standesrechts ist hier eine klare Grenze zu ziehen:

- Hausarzt und Spitalarzt müssen selbstverständlich nicht alle ihre Patienten routinemässig danach fragen, ob sie reglementierten Wettkampfsport betreiben ${ }^{12}$;

- Hingegen ist vom Sportmediziner und in den auf Sport spezialisierten Kliniken zu verlangen, dass sie routinemässig die Frage nach reglementiertem Wettkampfsport stellen.

Es geht zusammenfassend nicht um eine neue Berufsethik mit geänderten normativen Lösungen (du musst/darfst nicht), dies sei mit Nachdruck festgehalten. Nach wie vor bleibt die ärztliche Standesordnung verbindlich auch für sportärztliches Handeln. Nachdem nun aber das Strafrecht in einen Teilbereich dieses Handelns eingreift, galt es, dazu Interpretations- und Entscheidhilfen zu liefern.

Die Arbeitsgruppe hofft, dass mit dieser Haltung, wie sie vom Artikel 33bis der Standesordnung und der ergänzenden Richtlinie vertreten wird, den von Sportpatienten konsultierten Ärztinnen und Ärzten, seien sie nun in einem Team oder privat tätig, hilfreiche, gut verständliche und akzeptable Verhaltensempfehlungen angeboten werden, die sie weder mit ihrem Auftrag, Leiden zu mildern, noch mit dem Gebot, niemals zu schaden, in Konflikt geraten lassen. 


\section{Benützte Literatur (chronologisch)}

\section{A) Allgemein}

Verordnungsentwurf über die Dopingmittel und Methoden.

Bundesgesetz zur Förderung von Turnen und Sport: Massnahmen gegen das Doping, Art. 11c. (Siehe auch: www.dopinginfo.ch; medien; Hintergrundinformationen).

Dopingstatut und Dopingliste des SOV 2000.

Conférence Internationale des Ordres et des Organismes D'attributions similaires, Déclaration sur le Dopage dans les pratiques sportives, 15. Mai 2000.

Deklaration des Weltärztebundes über Grundsätze für die Gesundheitsfürsorge in der Sportmedizin, verabschiedet von der 34. Generalversammlung des Weltärztebundes Lissabon, Portugal, September/Oktober 1981 und revidiert von der (39., 45., und) 51. Generalversammlung des Weltärztebundes, Tel Aviv, Israel, Oktober 1999.

Code Antidopage du Mouvement Olympique, Chapitre II, Art. 1 et 2, p. 13, Lausanne 1999.

Guidelines for Physicians concerning sports medicine treatment, VSG, Netherlands Association of Sports Medicine, 1998

M. Kamber, B. Marti: Sport-seitige Nachfrage von Dopingmitteln - Dilemma in der Arztpraxis); Schweiz. Ärztezeitung 1998;46:2355-60.

Fédération Internationale de Médecine du Sport, Code of Ethics, 23. September 1997.

Dopinguntersuchungskommission (DUK), Schlussbericht zuhanden von «Sport Schweiz», 1995.

Stellungnahme der SGSM zur Dopingbekämpfung, La Chaux de Fonds, 30. September 1994.

Barry J. Maron, Robert W. Brown, Christopher A. McGrew, Matthew J. Mitten, Arthur L. Caplan, Adolph M. Hutter jun: Ethical, legal, and practical considerations impacting medical decision-making in competitive athletes (panel discussion); Official Journal of the American College of Sports Medicine, 1994;230-37.

Julius Sim, Coventry: Sports medicine: some ethical issues; Br J Sp Med 1993;27(2):95-100.

Bundesbeschluss über die Europäische Konvention gegen Doping vom 22. September 1992. Art. 2 (1) b).

\section{B) Spezifisch rechtliche Aspekte}

Jochen Fritzweiler (Hrsg,.): Doping; Verlage Stämpfli, Beck und Manz, 2000 (Bern, München und Wien).

Röhricht/Vieweg (Hrsg.); Doping-Forum: Aktuelle rechtliche und medizinische Aspekte; Richard Boorberg Verlag, 2000 (Deutschland).

Assessorin Mirja Kerstin Trautmann: Verschreibung von Anabolika trotz fehlender medizinischer Indikation (Kammergericht Baden Württemberg, Entscheid vom 3.3.1999); MedR 2/2000: 105-6. (Deutschland).

J. Y. Nau: Les bases éthiques de la lutte contre le dopage sportif; Médecine \& Hygiène 15.12.1999; 2486.
Prof. en droit C.-N. Robert, Genève: Dopage : que punir en priorité?; Médecine \& Hygiène 24.3.1999; 673-76.

Avocat et notaire François Vouillou, D.E.S (Genève), Sion: Règles de droit et règles de jeu en droit du sport l'exemple du dopage; Aktuelle Juristische Praxis (AJP) 2/1999; 161-6.

Prof. Dr. iur. Rainer Schröder und wiss. Mitarbeiterin Maren Bedau, Berlin: Doping: Zivilrechtliche Ansprüche des Konkurrenten gegen den gedopten Sportler, Neue Juristische Wochenschrift (NJW, Deutschland) 46/1999; 3361-67.

Prof. Dr. iur. W. Wiegand: Die Aufklärungspflicht; in H. Honsell (Hersg.): Handbuch des Arztrechts; Schulthess Verlag Zürich 1994; 119-213; betreffend Urteilsfähigkeit von Kindern und Jugendlichen: S. 159.

Dr. iur. Alexander Faber: Doping als unlauterer Wettbewerb und Spielbetrug; Diss. Zürich 1974 (Schulthess).

C) Um uns einen Überblick über die nationalen Richtlinien zu verschaffen, haben wir folgende Länder angefragt:

Schweden, Belgien, Dänemark, Australien, Spanien, Finnland, Frankreich, Grossbritannien, Norwegen, Deutschland, Österreich (jeweils Ärztegesellschaft oder falls vorhanden: medizinethische Kommission). Antworten haben wir aus folgenden Ländern erhalten:

Schweden hat zwei kurze Sätze in der «Standesordnung»: Ärzte sollen sich Methoden, die gegen med. Ethik, Wissenschaft oder Erfahrung gehen, widersetzen. Es ist deshalb gegen die medizinische Ethik, irgendeine Form von Doping anzuwenden.

Belgien: Keine direkten Weisungen betreffend Doping.

Finnland: Seit 1977 gilt: No drug shall be used in order to improve the prestation of healthy persons in sports, and doctors should not prescribe or recommend drugs for such purposes.

Australien verweist auf Australian Medical Association Position statement «Drugs in Sport».

Österreich: Broschüre: Antidoping. Keine konkreten ärztlichen Richtlinien.

Deutschland: verweist auf Weltärztebund-Deklaration Tel Aviv, Okt. 1999.

Great Britain: The GMC Standards Committee currently hold the view that Doctors who prescribe or collude in the provision of drugs or treatment with the intention of improperly enhancing an individual's performance in sport would be contravening the GMC's guidance, and such actions would usually raise a question over a doctor's continued registration. This does not preclude the provision of any care or treatment where the doctor's intention is to protect or improve the patient's health.

Norwegen: Keine Dopingregeln für Ärzte. Verweis auf Weltärztebund.

Holland: Broschüre: Guidelines for Physicians concerning sports medicine treatment, 1998. 